Ciência Florestal, Santa Maria, v. 22, n. 4, p. 813-820, out.-dez., 2012

ISSN 0103-9954

\title{
DOSES DE FÓSFORO DETERMINAM A PREVALÊNCIA DE FUNGOS MICORRÍZICOS ARBUSCULARES EM Araucaria angustifolia
}

\author{
PHOSPHORUS DOSES DETERMINE THE PREVALENCE OF NATIVE ARBUSCULAR \\ MYCORRHIZAL FUNGI IN Araucaria angustifolia
}

\author{
Milene Moreira $^{1}$ Dilmar Baretta ${ }^{2}$ Elke Jurandy Bran Nogueira Cardoso ${ }^{3}$
}

\begin{abstract}
RESUMO
Com o objetivo de avaliar o efeito de doses crescentes de fósforo sobre a diversidade, esporulação e colonização de fungos micorrízicos arbusculares (FMA) nativos de florestas de Araucaria angustifolia, foi conduzido um experimento em casa de vegetação, utilizando a araucária como planta-isca. O experimento foi montado em delineamento inteiramente casualizado, com cinco repetições, compreendendo quatro doses de fósforo $\left(0,20,50\right.$ e $\left.150 \mathrm{mg} \mathrm{kg}^{-1}\right)$ de substrato na forma de $\mathrm{KH}_{2} \mathrm{PO}_{4}$. As plântulas de araucária, inoculadas com $100 \mathrm{~g}$ de solo rizosférico, proveniente de mata nativa, foram cultivadas em vasos contendo $10 \mathrm{~kg}$ de solo, pelo período de um ano. Após a colheita, determinou-se a densidade de esporos, a taxa de colonização micorrízica e a diversidade de FMA, através da identificação morfológica dos esporos. Os dados foram submetidos à análise univariada e análise de correspondência (AC). Considerando as quatro doses de $\mathrm{P}$, foram identificadas 11 espécies de FMA (Acaulospora bireticulata, Acaulospora morrowiae, Acaulospora sp., Entrophospora colombiana, Gigaspora margarita, Glomus diaphanum, Glomus etunicatum, Glomus macrocarpum, Scutellospora calospora, Scutellospora gilmorei e Scutellospora pellucida). Não houve efeito significativo da dose de $\mathrm{P}$ sobre o número de esporos e a colonização radicular. A AC demonstrou que houve efeito da dose de P sobre a abundância de esporulação de cada espécie, sendo que alguns FMA se associaram mais com determinada dose de $\mathrm{P}$, e outros com outra.
\end{abstract}

Palavras-chave: FMA; diversidade; densidade de esporos; pinheiro brasileiro.

\begin{abstract}
A greenhouse experiment was installed with bait cultures to extract the AMF species present in a rhizosphere soil sample of a native Araucaria angustifolia forest in Campos do Jordão, Brazil. The experimental design was completely randomized, with four increasing phosphorus doses $\left(0,20,50\right.$, and $150 \mathrm{mg} \mathrm{kg}^{-1}$, as triple superphosphate), with five replicates, the bait plant was araucaria, and all pots were inoculated with $100 \mathrm{~g}$ of rhizospheric soil collected in an araucaria forest. After twelve months the spores were extracted, counted and identified, and the percent root colonization was also determined. When taking all four P doses into account, eleven AMF species could be identified: Acaulospora bireticulata, Acaulospora morrowiae, Acaulospora sp., Entrophospora colombiana, Gigaspora margarita, Glomus diaphanum, Glomus etunicatum, Glomus macrocarpum, Scutellospora calospora, Scutellospora gilmorei, and Scutellospora pellucida. There was no effect of the $\mathrm{P}$ dose on the total amount of spores neither on the percent root colonization. However, the correspondence analysis showed that the different AMF species were selectively associated mostly to either one or another P dose.
\end{abstract}

Keywords: AMF; diversity; spore density; Brazil Pine.

1. Engenheira Agrônoma, Dra ${ }^{\mathrm{a}}$, Pesquisadora Científica da Agência Paulista de Tecnologia dos Agronegócios, Pólo Regional Centro Sul, Caixa Postal 28, CEP 13400-970, Piracicaba (SP). mmoreira@apta.sp.gov.br

2. Engenheiro Agrônomo, Dr., Professor Efetivo do Centro de Educação Superior do Oeste (CEO/UDESC), Rua Beloni Trombeta Zanin, 680 E, CEP 89.815-630, Chapecó (SC).dilmar.baretta@udesc.br

3. Engenheira Agrônoma, Dra ${ }^{a}$., Professora Titular da Universidade São Paulo, Departamento de Ciência do Solo, Caixa Postal 09, CEP 13418-900, Piracicaba (SP). ejbncard@usp.br

Recebido para publicação em 5/10/2009 e aceito em 24/06/2011

Ci. Fl., v. 22, n. 4, out.-dez., 2012 


\section{INTRODUÇÃO}

Os fungos micorrízicos arbusculares (FMA) representam um dos maiores componentes da microbiota dos solos na maioria dos ecossistemas. São simbiontes obrigatórios com quase $90 \%$ das plantas superiores e pertencem ao filo Glomeromycota (SCHÜßLER et al., 2001).

As características químicas do solo podem ser determinantes para a distribuição dos FMA nos diversos ecossistemas, onde o predomínio de certas espécies pode ocorrer. As alterações no $\mathrm{pH}$ do solo influenciam a ocorrência das espécies dos FMA e a densidade dos esporos no solo (TRUFEM, 1990). $\mathrm{O}$ pH do solo influencia a associação micorrízica, porque pode reduzir a germinação dos esporos e o crescimento do tubo germinativo (SILVEIRA, 1998). Os valores baixos de $\mathrm{pH}$ favorecem as espécies de Acaulospora, Gigaspora e Scutellospora, enquanto que as espécies de Glomus são favorecidas por $\mathrm{pH}$ mais próximo da neutralidade (SIQUEIRA et al., 1989; TRUFEM, 1990; SILVEIRA, 1998).

A disponibilidade de fósforo no solo também pode afetar a eficiência de algumas espécies de fungos MA, influenciando na colonização das raízes e na composição e distribuição das espécies de fungos no ambiente (PICONE, 2000). A densidade de esporos e hifas nos solos pobres em $\mathrm{P}$ pode ter ligeiro incremento com aplicações moderadas de P (5-15 kg P ha-1) (THOMSON et al., 1992) em contrapartida, altas aplicações de $\mathrm{P}$ podem causar um decréscimo na densidade de esporos e hifas. Esse comportamento pode ser frequentemente observado em diferentes solos e condições climáticas (DOUDS e MILLNER, 1999; KAHILUOTO et al., 2001).

Os propágulos de FMA, recolhidos em campo, podem ser multiplicados em vasos em associação com plantas micotróficas, e esse processo é conhecido como iscagem ou cultura-armadilha (STUTZ e MORTON, 1996). As espécies que esporulam nessa condição têm maiores chances de serem identificadas. Todavia, vários fatores, como duração do ciclo de vida das plantas hospedeiras, tipo de substrato, $\mathrm{pH}$, disponibilidade de $\mathrm{P}$, outros nutrientes e a temperatura, influenciam diretamente os resultados relativos à composição de espécies em comunidades de FMA (SIEVERDING, 1991; MORTON et al., 1993; BEVER et al., 2001; MOREIRA et al., 2007b). Neste contexto, o presente estudo teve como objetivo avaliar o efeito de doses crescentes de fósforo sobre a diversidade, riqueza, esporulação e colonização micorrízica dos
FMA provenientes de floresta nativa de Araucaria angustifolia (Bertoloni) Otto Kuntze, localizada no município de Campos do Jordão, SP, junto à sua planta hospedeira, em vasos.

\section{MATERIAL E MÉTODOS}

Os fungos micorrízicos nativos utilizados no presente estudo foram provenientes de amostras de floresta nativa de Araucaria angustifolia localizada no município de Campos do Jordão, SP, (contendo os gêneros Glomus, Acaulospora, Gigaspora, Entrophospora e Scutellospora). Mais informações sobre a área de floresta nativa de Araucária, onde foram coletadas as espécies de FMA, podem ser obtidas em Moreira-Souza et al. (2003).

O substrato utilizado no experimento de casa de vegetação foi proveniente de uma amostra de Neossolo Quartzarênico (série Paredão Vermelho) + areia lavada de rio na proporção de 2:1 (V:V), com as seguintes características: $\mathrm{pH}$ em $\mathrm{CaCl}_{2} 3,9 ; 14 \mathrm{~g} \mathrm{dm}^{-3}$ de matéria orgânica; $2 \mathrm{mg} \mathrm{dm}^{-3}$ de P (resina); 0,$5 ; 3 ; 1$ e $47 \mathrm{mmol}_{\mathrm{c}} \mathrm{dm}^{-3} \mathrm{de} \mathrm{K}^{+}, \mathrm{Ca}^{+2}$, $\mathrm{Mg}^{+2}$ e $\mathrm{H}^{+}+\mathrm{Al}^{+3}$, respectivamente.

A mistura foi autoclavada por duas horas a $121^{\circ} \mathrm{C}$. Adicionaram-se 5,5 g de calcário dolomítico PRNT $131 \%$ em cada vaso de $20 \mathrm{~L}$, elevando o pH para 6,0 e foi feita incubação por 20 dias (MOREIRA-SOUZA e CARDOSO, 2002). Posteriormente, foi adicionado o $\mathrm{P}$, nas quatro doses previstas para os diferentes tratamentos $0,20,50$ e $150 \mathrm{mg} \mathrm{kg}^{-1}$ de substrato na forma de $\mathrm{KH}_{2} \mathrm{PO}_{4}$ e incorporaram-se $100 \mathrm{~g}$ de solo-inóculo quando as plântulas foram transferidas para os vasos. Adicionalmente, foi colocada solução nutritiva de Hoagland (HOAGLAND e ARNON, 1938) isenta de $\mathrm{P}$ a cada dois meses, durante a condução do experimento. $\mathrm{O}$ experimento foi conduzido em delineamento inteiramente casualizado, com cinco repetições, no Departamento de Ciência do Solo da ESALQ/USP, Piracicaba, SP, instalado no mês de junho.

As sementes de Araucaria angustifolia, também provenientes do Parque Estadual de Campos do Jordão, foram selecionadas, desinfestadas em hipoclorito de sódio a $0,5 \%$, escarificadas e semeadas em caixas com areia de rio esterilizada (MOREIRA-SOUZA e CARDOSO, 2003b).

As plantas de araucária foram utilizadas como cultura-armadilha e foram mantidas em casa de vegetação por 12 meses. Os esporos foram extraídos de $50 \mathrm{~g}$ de solo seco desses vasos para contagem 
e identificação morfológica de espécies de FMA. As raízes foram coloridas conforme VIERHEILIG et al. (1998) e determinou-se a percentagem de colonização radicular (GIOVANETTI e MOSSE, 1980).

Para a identificação, os esporos foram separados em grupos de acordo com as características morfológicas e montadas lâminas semipermanentes com PVLG e reagente de Melzer (MORTON et al., 1993). Posteriormente, as lâminas foram observadas em microscópio ótico, sendo identificados os esporos com auxílio do manual de Schenck e Pérez (1990) e das descrições fornecidas pelo INVAM (2004).

A abundância de esporos de FMA e a colonização radicular foram submetidas à análise de variância (ANOVA), usando o programa SAS versão 8.2 (SAS, 1996), e as médias comparadas pelo teste LSD $(P<0,05)$. A abundância de esporos de FMA foi utilizada para a obtenção do comprimento do gradiente (DCA) (ter BRAAK e SMILAUER, 1998) para proceder à análise multivariada. Como o comprimento do gradiente para abundância de esporos de FMA foi maior do que três (resposta não linear), optou-se pela análise de correspondência (AC), usando o programa CANOCO versão 4.0 (ter BRAAK, 1986; ter BRAAK e SMILAUER, 1998).

\section{RESULTADOS}

O solo da região das raízes das araucárias nativas, utilizado como inóculo nesse experimento, continha 24 espécies de FMA, sendo oito espécies de Acaulospora, uma de Entrophospora, duas de Gigaspora, nove de Glomus e quatro de Scutellospora (MOREIRA-SOUZA et al., 2003a). Após o período de crescimento das araucárias-iscas inoculadas com este material, foram identificadas apenas 11 espécies de FMA e uma média de 603 esporos em $50 \mathrm{~g}$ de solo, considerando-se as quatro doses de P (Tabela 1).

A análise de variância (ANOVA) para o número de esporos e para a colonização radicular das plantas de araucária indicou que não houve efeito significativo da dose de $\mathrm{P}$ sobre estes parâmetros (Figura 1).

A identificação morfológica dos esporos encontrados em cada dose de P pode ser visualizada na Tabela 1. A presença ou ausência de determinada espécie de FMA nas doses de P foi bastante variável, aparentemente não apresentando uma regra lógica. Quando estes resultados foram submetidos à $\mathrm{AC}$, foi possível observar que havia certa analogia entre a dose de $\mathrm{P}$ no solo e as espécies que se destacavam em cada uma delas.

TABELA 1: Número de esporos de fungos micorrízicos arbusculares (FMA) em diferentes níveis de P em $50 \mathrm{~g}$ de solo da floresta de Araucaria angustifolia, em Campos do Jordão, SP, Brasil. ( $\mathrm{n}=5)$.

TABLE 1: Number of spores of AMF in different P levels. Campos do Jordão, SP state, Brazil. ( $\mathrm{n}=5$ ).

\begin{tabular}{|c|c|c|c|c|c|}
\hline \multirow[t]{2}{*}{ Espécies de fungos micorrízicos arbusculares } & \multicolumn{5}{|c|}{ Doses de fósforo $(\mathrm{P})$} \\
\hline & Abreviação & 0 & 20 & 50 & 150 \\
\hline Acaulospora bireticulata Rothwell \& Trappe & (A.biret) & $-(1)$ & - & - & 02 \\
\hline Acaulospora morrowiae Spain \& Schenck & (A. morr) & - & 57 & 75 & 10 \\
\hline Acaulospora sp. & $($ A.sp 1$)$ & 105 & 02 & 24 & 89 \\
\hline Entrophospora colombiana Spain \& Schenck & $($ E.col $)$ & 02 & 62 & - & 01 \\
\hline Gigaspora margarita Becker \& Hall & (G.marg) & - & 09 & 01 & - \\
\hline Glomus diaphanum Morton \& Walker & (Gl.diaph) & - & 05 & - & - \\
\hline Glomus etunicatum Becker \& Gerd. & (Gl.etun) & - & 10 & - & - \\
\hline Glomus macrocarpum Tul. \& Tul. & (Gl.macr) & 19 & 06 & 09 & 02 \\
\hline Scutellospora calospora (Nicolson \& Gerd.) Walker \& Sanders & $($ S.calo $)$ & - & 15 & 03 & 28 \\
\hline Scutellospora gilmorei (Trappe \& Gerd.) Walker \& Sanders & $($ S.gilm $)$ & 01 & - & - & - \\
\hline Scutellospora pellucida (Nicol. \& Schenck) Walker \& Sanders & (S.pell) & 14 & 05 & 13 & 34 \\
\hline Número total de esporos de FMA & (Tspo) & 141 & 171 & 125 & 166 \\
\hline
\end{tabular}

Em que: ${ }^{(1)}=$ Ausência de esporos; ${ }^{(1)}=$ no spores detected. 
(A)

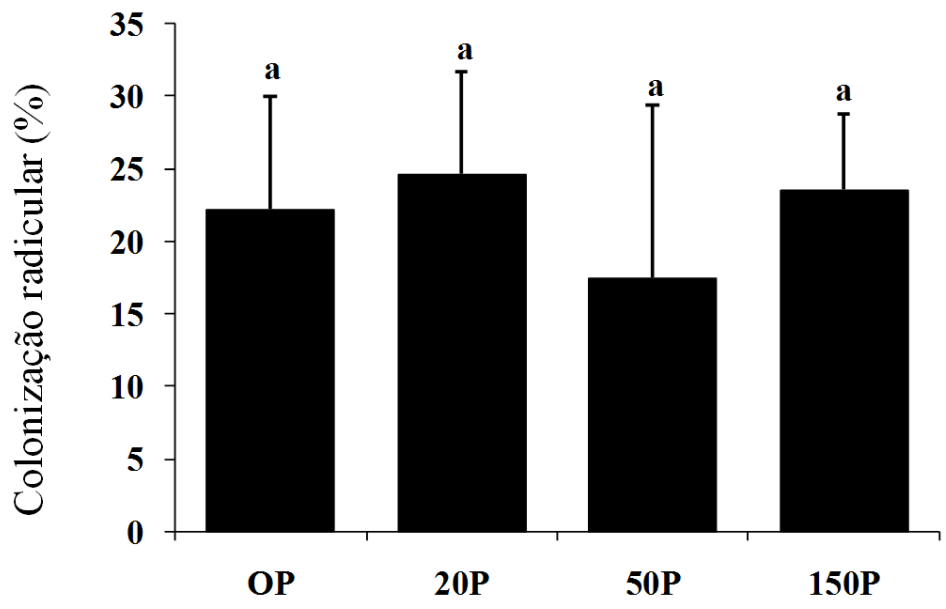

(B)

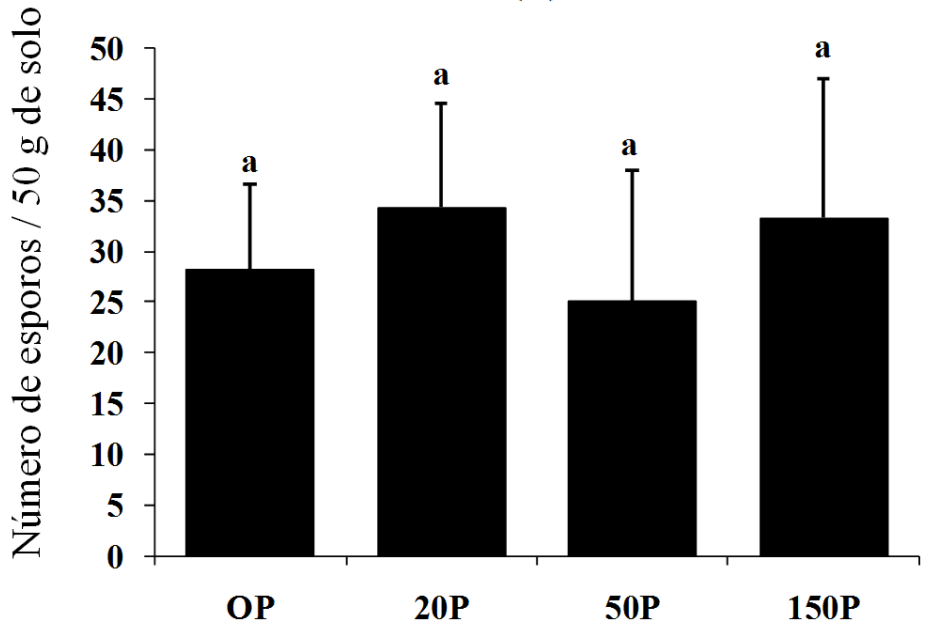

FIGURA 1: Colonização radicular por fungos micorrízicos arbusculares (A) e número de esporos (B) em quatro doses de fósforo (P): zero (0P), 20 (20P), 50 (50P) e 150 (150P) $\mathrm{mg} \mathrm{kg}^{-1}$ de solo. Média de 5 repetições. $(T)$ Desvios padrão. Médias com a mesma letra não diferem estatisticamente $(P<0,05)$ pelo teste LSD.

FIGURE 1: Root colonization rate by arbuscular mycorrhizal fungi (A) and Number of spores (B) in (OP), $20(20 \mathrm{P}), 50(50 \mathrm{P})$ e $150(150 \mathrm{P}) \mathrm{mg} \mathrm{kg}^{-1}$ phosphorus P levels. Mean of five replicates. $(\mathrm{T})$ Standard deviation. Means followed by the same letters do not differ $(P<0.05)$ by the LSD test.

Constatou-se que na dose zero, onde não foi adicionado $\mathrm{P}$, houve esporulação de cinco espécies de FMA (Acaulospora sp., Entrophospora colombiana, Glomus macrocarpum, Scutellospora gilmorei e Scutellospora pellucida), sendo a maior abundância a de Acaulospora sp. A espécie Scutellospora pellucida foi encontrada em todas as doses de $\mathrm{P}$, embora com maior número na maior dose. Na dose $20 \mathrm{mg} \mathrm{kg}^{-1}$ de $\mathrm{P}$, foram encontradas nove espécies de FMA (Acaulospora morrowiae, Acaulospora sp, Entrophospora colombiana, Gigaspora margarita, Glomus diaphanum, Glomus etunicatum, Glomus macrocarpum, Scutellospora calospora e Scutellospora pellucida), sendo as mais abundantes Entrophospora colombiana e Acaulospora morrowiae. As espécies Glomus diaphanum e Glomus etunicatum somente foram encontradas nessa dose. $\mathrm{Na}$ dose $50 \mathrm{mg} \mathrm{kg}^{-1}$ de $\mathrm{P}$, foram encontradas seis espécies de FMA (Acaulospora morrowiae, Acaulospora sp., Gigaspora margarita, Glomus macrocarpum, Scutellospora calospora e Scutellospora pellucida), com predominância de Acaulospora morrowiae e Acaulospora sp. Entretanto, na maior dose de $\mathrm{P}$ 
(150 mg kg-1 de P) esporularam sete espécies de FMA (Acaulospora bireticulata, Acaulospora morrowiae, Acaulospora sp., Entrophospora colombiana, Glomus macrocarpum, Scutellospora calospora e Scutellospora pellucida), sendo Acaulospora sp. a mais abundante; Acaulospora bireticulata somente foi encontrada neste tratamento.

\section{Análise de Correspondência (AC)}

A associação das diferentes espécies de FMA com as quatro doses de $\mathrm{P}\left(0,20,50\right.$ e $\left.150 \mathrm{mg} \mathrm{kg}^{-1}\right)$ está apresentada num gráfico de ordenação (Figura1), com eixos perpendiculares (Dimensões 1 e 2), que representam a variação multidimensional de um conjunto de variáveis de resposta (abundância de espécies de FMA) nas doses de P (tratamentos) (ter BRAAK, 1986; MOREIRA et al., 2009).

Esta análise forneceu uma nítida separação entre as doses de $\mathrm{P}$ e as espécies de FMA que mais foram influenciadas por cada dose. As dimensões 1 e 2 corresponderam a $34,5 \%$ e $18,5 \%$ da variabilidade, respectivamente, explicando em conjunto $53 \%$ da variabilidade total dos dados.

De modo geral, as doses de $\mathrm{P}$ ficaram dispostas em quadrantes separados, assim como, também, as diferentes espécies, de acordo com a dose que mais influenciou na abundância de cada espécie de FMA (Figura 2). Ao longo da dimensão 1 (eixo principal), o tratamento $20 \mathrm{P}$ está disposto no quadrante direito, enquanto que os tratamentos 50P e 150P ficaram muito próximos um do outro e dispostos na porção inferior esquerda (com valores negativos nas dimensões 1 e 2), demonstrando que esses dois últimos tratamentos são muito similares em termos de composição de espécies de FMA.

\section{DISCUSSÃO}

A pequena diversidade de FMA isolada por culturas-armadilhas em comparação com o número inicialmente detectado em campo sugere que este método pode subestimar a verdadeira abundância presente no solo de origem, fato que já foi discutido detalhadamente em Moreira et al. (2007b).

$\mathrm{Na}$ Figura 2, quanto mais próxima a um determinado valor de $\mathrm{P}$ estiver localizada determinada espécie de FMA, maior é a influência desse valor na abundância desta espécie. Nesse sentido, as espécies Acaulospora bireticulata, Scutellospora pellucida e Acaulospora morrowiae apareceram mais ligadas às duas maiores doses de $\mathrm{P}(50 \mathrm{P}$ e $150 \mathrm{P})$, pois devem ser mais tolerantes a doses elevadas ou, talvez, até sejam favorecidas em solos com maiores teores desse elemento. As espécies Acaulospora sp. e Glomus macrocarpum, assim como Scutellospora gilmorei, foram estimuladas a esporular no tratamento sem fósforo adicional (0P). Contudo, as demais espécies de FMA (Glomus etunicatum, Gigaspora margarita, Entrophospora colombiana e Glomus diaphanum) esporularam mais no tratamento com dose baixa de $\mathrm{P}$ (20P). É interessante que a dose de $20 \mathrm{mg} \mathrm{kg}^{-1}$ de P mostrou-se como a mais favorável para Gigaspora margarita, visto que nessa concentração de P este endófito mostrou sua maior eficiência micorrízica em Araucaria angustifolia, em estudo anterior sobre o micotrofismo dessa planta (MOREIRA-SOUZA e CARDOSO, 2002).

$\mathrm{O}$ número total de esporos de FMA e a percentagem de colonização radicular não se associaram a nenhum dos tratamentos, uma vez que estão dispostos na porção mediana da Figura 2. Este resultado apenas corrobora o achado, evidenciado pela análise estatística univariada, ou seja, nas condições desse estudo, a dose de $\mathrm{P}$ não afetou a esporulação total dos FMA ou a colonização radicular da araucária.

Os números de esporos encontrados no presente estudo foram menores que os obtidos por MOREIRA et al. (2006) e MOREIRA et al. (2007a), que registraram mais que 1000 esporos por $100 \mathrm{~g}$ de solo seco de FMA em áreas de florestas com Araucaria angustifolia em Campos do Jordão e Apiaí, SP. No entanto, o menor número de esporos e de diversidade de espécies de FMA encontrados na casa de vegetação, em comparação com amostras de campo coletadas em áreas com Araucaria angustifolia, pode ser resultado de uma série de fatores, como: tamanho do vaso, temperatura, idade da planta, doses de $\mathrm{P}$, luminosidade, umidade, entre outros. As florestas nativas de araucária, impactadas ou não pela ação antrópica, de uma maneira geral, possuem maior heterogeneidade de hábitat e são capazes de suportar maior diversidade de espécies de FMA (MOREIRA-SOUZA et al., 2003a; MOREIRA et al., 2006; MOREIRA et al., 2007a).

A quantidade total de esporos de FMA não foi afetada pelas doses crescentes de P nesse estudo de esporulação junto ao hospedeiro-armadilha; no entanto, na literatura, pode ser encontrada grande divergência de um caso para outro, conforme já foi relatado há mais de vinte anos (SCHENCK e SMITH, 1982). Em alguns trabalhos de campo ou de casa de vegetação, a produção de esporos 


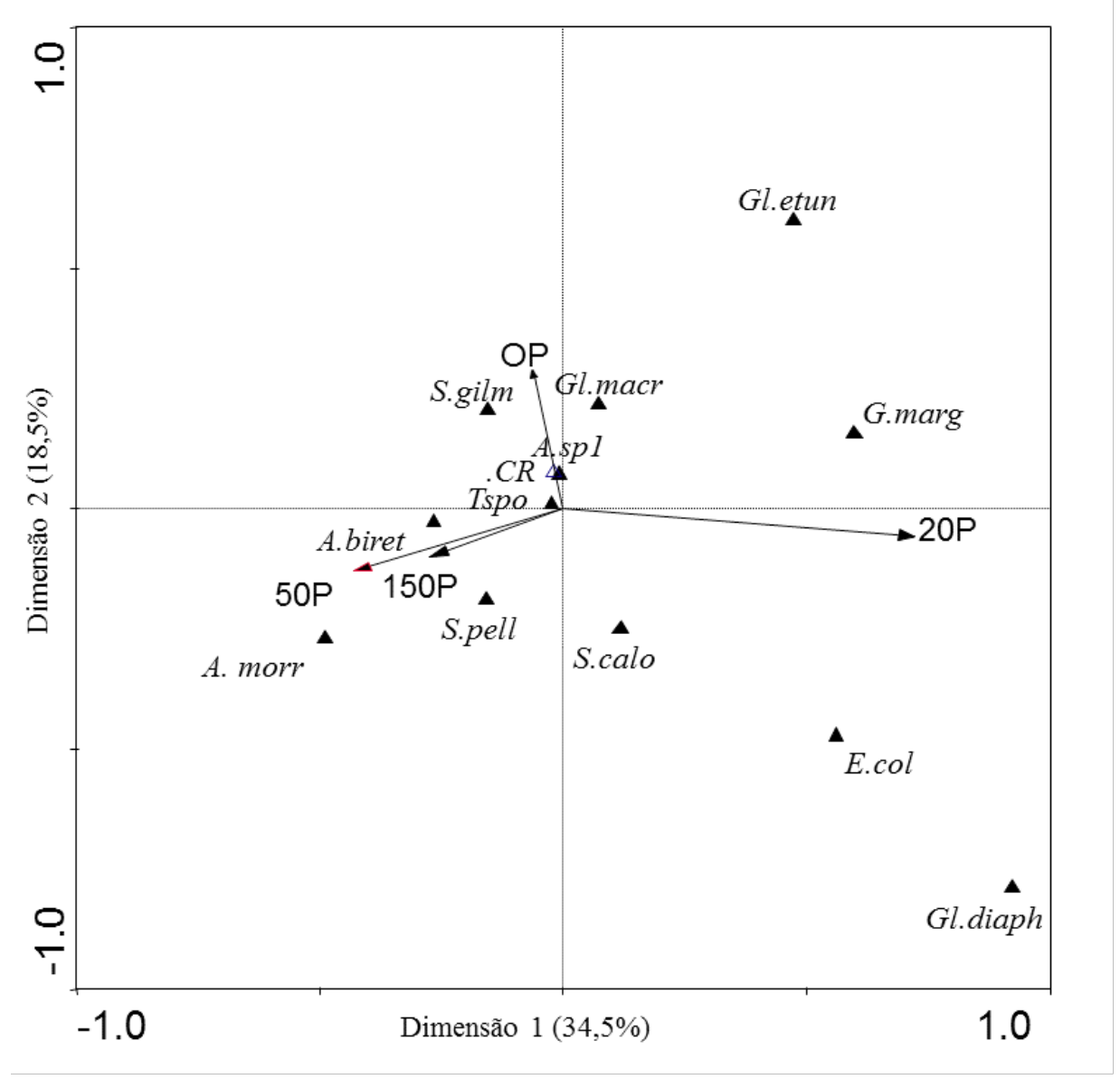

FIGURA 2: Relação entre dimensões 1 e 2 da análise de correspondência (CA) para as doses zero (0P), 20 (20P), $50(50 \mathrm{P})$ e 150 (150P) $\mathrm{mg} \mathrm{kg}^{-1}$ de $\mathrm{P}$ e espécies de FMA $(\mathrm{n}=5)$. Campos do Jordão, SP. A. biret $=$ Acaulospora bireticulata; A. morr $=$ A. morrowiae; A.sp $1=$ Acaulospora sp. 1 ; E.col = Entrophospora colombiana; G. marg = Gigaspora margarita $;$ Gl.diaph $=$ Glomus diaphanum; Gl.etun $=$ G. etunicatum; Gl.macr $=$ G. macrocarpum; S.calo $=$ Scutellospora calospora; S.gilm $=$ S. gilmorei; S.pell $=$ S. pellucida; Tspo $=$ Total número de esporos de FMA; $C R=$ colonização radicular (\%).

FIGURE 2: Relation between dimensions 1 and 2 of the correspondence analysis (CA) for doses of zero (OP), 20 (20P), 50 (50P) and $150(150 \mathrm{P}) \mathrm{mg} \mathrm{kg}^{-1}$ de $\mathrm{P}$ and AMF species $(\mathrm{n}=5)$. Campos do Jordão, SP state, 1998. A. biret = Acaulospora bireticulata; A. morr $=$ A. morrowiae; A.sp 1 =Acaulospora sp.1; E.col = Entrophospora colombiana; G. marg = Gigaspora margarita; Gl.diaph $=$ Glomus diaphanum; Gl.etun $=$ G. etunicatum; Gl.macr $=$ G. macrocarpum; S.calo $=$ Scutellospora calospora $;$ S.gilm $=$ S. gilmorei; S.pell $=$ S. pellucida $;$ Tspo $=$ Total number of AMF spores; $C R=$ Root colonization (\%).

é influenciada positivamente pelo incremento de $\mathrm{P}$, em outros a influência é negativa (EZETA e SANTOS, 1980; NEUMANN e GEORGE, 2004; SUBRAMANIAN et al., 2006), ou ainda, não ocorre influência na esporulação (TCHABI et al., 2008). Assim, o aumento de P disponível no solo diminuiu a abundância de esporos de Scutellospora heterogama e não interferiu na de Glomus spp. (de MIRANDA e HARRIS, 1994), e a espécie Glomus macrocarpum mostrou maior esporulação nas maiores doses de fosfato (ROSS e HARPER, 1970). - A colonização radicular, frequentemente, é inversamente relacionada ao suprimento de $\mathrm{P}$, embora pequenas adições de $\mathrm{P}$ a solos deficientes possam elevar a colonização. No entanto, no presente estudo as doses crescentes de P não demonstraram efeito sobre esta variável. A colonização das plantas de araucária com FMA nativos foi similar a de outros estudos (MOREIRA-SOUZA e CARDOSO, 2002; MOREIRA-SOUZA et al., 2003a; MOREIRA et al., 
2006; MOREIRA et al., 2007a e 2007b), em que os valores variam de 20 a $25 \%$. Observou-se, também, que nesse e nos outros estudos a concentração de $\mathrm{P}$, mesmo na dose zero (quantidade natural de $\mathrm{P}$ no solo), foi suficiente para o bom desenvolvimento das plantas. Entretanto, já é bem conhecido que nem sempre existe relação direta entre o grau de colonização radicular e a eficiência micorrízica (GRAHAM et al., 1982).

\section{CONCLUSÕES}

A colonização radicular e o número total de esporos de FMA produzidos não foram afetados pelas doses crescentes de $\mathrm{P}$, em ensaio de vasos com araucária como planta-armadilha e com soloinóculo de FMA nativos.

Doses crescentes de $\mathrm{P}$ tiveram efeitos diferenciados na prevalência das diferentes espécies de FMA nativas de floresta de araucária, junto à sua planta hospedeira.

\section{AGRADECIMENTOS}

M. Moreira e D. Baretta agradecem à Fundação de Amparo à Pesquisa do Estado de São Paulo (FAPESP) pela bolsa de PósDoutorado (processo no. 01/13229-9 e processo no. 2007/05637-6, respectivamente). Agradecemos pelo suporte financeiro do projeto BIOTA/FAPESP (Processo 01/05146-6), do Programa Instituto Biodiversidade Virtual (www.biotasp.org.br) e ao IBAMA pela permissão da coleta do material (processo no. 02027.002353/05-94). E.J.B.N. Cardoso agradece ao Conselho Nacional de Pesquisa (CNPq) pela bolsa de produtividade. Agradecemos, também, à Denise Mescolotti e Luis Fernando Baldesin pelo auxílio técnico.

\section{REFERÊNCIAS BIBLIOGRÁFICAS}

BEVER, J. D. et al. Arbuscular mycorrhizal fungi: more diverse than meets the eye, and the ecological tale of why. Bioscience, v. 51, p. 923-931, 2001.

DE MIRANDA, J. C. C.; HARRIS, P. J. The effect of soil phosphorus on the external mycelium growth of arbuscular mycorrhizal fungi during the early stages of mycorrhizal formation. Plant and Soil, v. 166, p. 271-280, 1994.

DOUDS, D. D.; MILLNER, P. Biodiversity of arbuscular mycorrhizal fungi in agroecosystems. Agriculture, Ecosystems \& Environment, v.74, p.77-93, 1999.

EZETA, F. N.; SANTOS, O. M. Benefício da introdução de endomicorriza eficiente na utilização de nutrientes em latossolos do sul da Bahia, Revista Brasileira de Ciência do Solo, v. 4, p. 13-17, 1980. GIOVANNETTI, M.; MOSSE, B. An evaluation of techniques for measuring vesicular arbuscular mycorrhizal infection in roots. New Phytologist, v. 84, p.489-500, 1980.

GRAHAM, J. H et al. Development of external hyphae by different isolates of mycorrhizal Glomus spp. in relation to root colonisation and growth of Troyer citrange. New Phytologist, v. 91, p.183-189, 1982.

HOAGLAND, D. R.; ARANON, D. I. The waterculture method for growing plants without soil. California Agricultural Experimental Station. 1938.

INVAM (2004) International Culture Collection of Vesicular and Arbuscular Mycorrhizal Fungi. Species Description. Morgantown, West Virginia Agriculture and Forestry Experimental Station. Home page. [http: $\backslash \backslash$ invam.caf.wvu.edu]

KAHILUOTO, $\mathrm{H}$. et al. Promotion of AMF utilization through reduced $P$ fertilization 2. Field studies. Plant and Soil, v. 231, p. 65-79, 2001.

MOREIRA, M. et al. Arbuscular mycorrhizal fungal communities in native and in replanted Araucaria forest: a case study. Scientia Agricola, v. 66, p. 677-684, 2009.

MOREIRA, M. et al. Spore density and root colonization by arbuscular mycorrhizal fungi in preserved or disturbed Arancaria angustifolia (Bert.) O. Ktze. ecosystems. Scientia Agricola, v. 63, p. 380-385, 2006.

MOREIRA, M. et al. Sporulation and diversity of arbuscular mycorrhizal fungi in Brazil Pine in the field and in the greenhouse. Mycorrhiza, v. 17, p. 519-526, $2007 \mathrm{~b}$.

MOREIRA. M. et al. Biodiversity and distribution of arbuscular mycorrhizal fungi in Araucaria angustifolia forest. Scientia Agricola, v. 64, p. 393399, 2007a.

MOREIRA-SOUZA, M. et al. Arbuscular mycorrhizal fungi associated with Araucaria angustifolia (Bert.) O. Ktze. Mycorrhiza, v.13, p. 211-215, 2003a.

MOREIRA-SOUZA, M.; CARDOSO, E. J. B. N. Dependência micorrízica de Araucaria angustifolia (Bert.) O. Ktze. sob doses de fósforo. Revista Brasileira de Ciência do Solo, v. 26, p. 905-912, 2002. 
MOREIRA-SOUZA, M.; CARDOSO, E. J. B. N. Practical method for germination of Araucaria angustifolia (Bert.) O. Ktze. seeds. Scientia Agricola, v. 60, p. 389-391, 2003 b.

MORTON, J. B. et al. Germplasm in the international Collection of Arbuscular and Vesicular Arbuscular Mycorrhizal Fungi (INVAM) and procedures for culture development, documentation and storage. Mycotaxon, v. 48, p.491-528, 1993.

NEUMANN, E.; GEORGE, E. Colonisation with the arbuscular mycorrhizal fungus Glomus mosseae (Nicol. \& Gerd.) enhanced phosphorus uptake from dry soil in Sorghum bicolor (L.). Plant and Soil, v. 261, p. 245-255, 2004.

PICONE, C. Diversity and abundance of arbuscularmycorrhizal fungus spores in tropical forest and pasture. Biotropica, v. 32, p. 734-750, 2000.

ROSS, J. P.; HARPER, J. A. Effect of endogone mycorrhiza on soybean yields. Phytopathology, v.60, n. 1, p. 552-556, 1970.

SAS Institute. SAS/STAT. Release 6.2. Cary: SAS Institute Inc., 1996.

SCHENCK, N. C.; PÉREZ, Y. Manual for the identification of VA mycorrhizal fungi, 3rd ed. Gainesville: Synergistic, 1990.

SCHENCK, N. C.; SMITH, G. S Responses of six species of vesicular-arbuscular mycorrhizal fungi and their effects on soybean at four soil temperatures, New Phytologist, v. 92, n. 2, p.193-201, 1992.

SCHÜ $\beta L E R, A$. et al. Analysis of partial Glomales SSU rRNA gene sequence: implications for primer design and phylogeny. Mycology Research, v.105, p. 05-15, 2001.

SIEVERDING, E. Vesicular arbuscular mycorrhiza management in tropical agrosystems. Eschborn, Germany: GTZ, 1991. 371 p.

SILVEIRA, A. P. D. Ecologia de fungos micorrízicos arbusculares. In: MELO, J. L. (ed). Ecologia microbiana. Jaguariúna: Embrapa, 1998. p. 61-83.

SIQUEIRA, J. O. et al. Mycorrhizal colonization and mycotrophic growth of native woody species as related to successional groups in southeastern Brazil. Forest Ecology and Management, v. 107, p. 241-252, 1998.

STUTZ, J. C.; MORTON, J. B. Successive pot cultures reveal high species richness of arbuscular endomycorrhizal fungi in arid ecosystems, Canadian Journal Botany, v. 74, p. 1883-1889, 1996.

SUBRAMANIAN, K. S. et al. Response of field grown tomato plants to arbuscular mycorrhizal fungal colonization under varying intensities of drought stress. Scientia Horticulturae, v. 107, p. 245-253, 2006.

TCHABI, A. et al. Arbuscular mycorrhizal fungal communities in sub-Saharan savannas of Benin, West Africa, as affected by agricultural land use intensity and ecological zone. Mycorrhiza, v. 18, p. 181-195, 2008.

ter BRAAK, C. J. F. Canonical correspondence analysis: a new eigenvector technique for multivariate direct gradient analysis. Ecology, v. 67, p. 1167-1179, 1986.

ter BRAAK, C. J. F.; SMILAUER, P. CANOCO Reference Manual and User's Guide to Canoco for Windows: software for canonical community ordination (version 4). New York: Microcomputer Power. 1998.

THOMSON, B. D. et al. The effect of long-term applications of phosphorus fertilizer on populations of vesicular-arbuscular mycorrhizal fungi in pastures. Australian Journal of Agriculture Research, v. 43, p. 1131-1142, 1992.

TRUFEM, S. F. B. Aspectos ecológicos de fungos micorrízicos vesículo-arbusculares da mata tropical úmida da Ilha do Cardoso, SP, Brasil. Acta Botanica Brasilica, v. 4, n. 2, p. 31-45, 1990.

VIERHEILIG, H. et al. Ink and vinegar, a simple staining technique for arbuscular-mycorrhizal fungi. Applied and Environmental Microbiology, v. 64, p. 5004-5007, 1998. 